\title{
Ethical challenges in Research, Industry and People Partnerships in mental health
}

\author{
Sapfo Lignou*§, John Geddes*, Ilina Singh*
}

\section{Abstract}

Advancements in mental health research, social changes and policy developments have led to the emergence of new forms of research partnerships, which bring together research institutions, public companies and lay people as partners in the same research project. In this paper, we argue that partnerships comprised of industry, academia and people with experience of mental illness may present practical and ethical challenges that affect the conduct of research and undermine public trust in research collaborations. We outline a number of ethical problems from the motivation to combine competing interests and values of these diverse research partners. We argue that while critical perspectives on each of the partnership forms outlined above exist in the literature, the combination of industry, research and PPI actors in partnership in mental health research has not received sufficient scrutiny. We suggest that a robust ethical approach is needed to properly substantiate the value of such research partnerships, to inform practical and ethical guidance on potential conflicts and to facilitate productive collaborative research.

\section{Keywords}

Research partnerships, mental health, pharmaceutical companies, PPI, service users, academia, competing interests

\section{Introduction: Academia, Industry and people with lived experience of mental illness as 'partners' in mental health research}

Advancements in mental health research, social changes and policy developments have led to the emergence of new forms of research partnerships among stakeholders in mental health; specifically, research institutions, industry (pharmaceutical and biotech) and people with lived experience of mental health challenges. We call such research partnerships RIPs (Researchers, Industry and People). There are at least two types of RIPs in the European Union today: The first is illustrated by the EU Innovative Medicines Initiative (IMI), which is a publicprivate partnership between the European Union and the European pharmaceutical industry, with a $€ 3.3$ billion budget between 2014-2020. In this type of RIP, an alliance between industry and researchers drives the value of the funding mechanism and is transparently articulated and structured. The inclusion of a patients, carers and public stakeholders element (PPI), is far less clearly or equitably outlined, although it is strongly "encouraged" and advertised as a valued 'partnership'. As of 2019, IMI maintains its own pool of 'patient experts' that provides PPI guidance to researchers. ${ }^{1}$

A second type of RIP is sponsored by a public institution, in which RIP partnerships are seen to drive scientific, economic and public value. The UK's National Institute for Health Research (NIHR) is one such example, where working across academic institutions, third sector organisations and industry is seen to support a worldleading 'research ecosystem'. ${ }^{2}$ In this version of a RIP, the structure of mutual obligations among the three entities brought together in 'partnership' appears to change over time and perhaps according to the leadership of the moment. For example, in its current iteration, the NIHR places high value on the role of PPI in academic research, with absolute requirements for PPI involvement in research projects:

* Department of Psychiatry, University of Oxford

$\S$ Corresponding author. sapfo.lignou@psych.ox.ac.uk NEUROSEC, Department of Psychiatry and Wellcome Centre for Ethics and Humanities, University of Oxford 
"How you've involved patients, carers and the public will be assessed when your research application is reviewed by our prioritisation and funding committees" ${ }^{3}$

Involvement with industry, on the other hand, is only encouraged:

"You may wish to consider partnering or collaborating with the life sciences industry or charities." ${ }^{3}$

On the other hand, the NIHR actively solicits revenue from industry by offering the NHS as a research service platform for industry studies. ${ }^{4}$ NIHR's partnership services include PPI at the start of an industry-sponsored study, but it is unclear from publicly available materials whether NIHR requires PPI in partnered industrysponsored studies, or if it is only recommended as good practice ${ }^{5}$

Communications from governments, industry and academia advertise the value of such research partnerships, highlighting the positive potential for progress in mental health research and treatments, such as gains in efficiency, skills and resources ${ }^{6}$

"[We] hope, by working in partnership with patients, bringing in new talent, and joining up the resources we have already, that we can transform the landscape of mental health research-and by doing so to improve the lives of those affected")'?

Such positioning of national strategy veils core challenges to this partnership agenda that threaten its very success. In what follows, we outline a number of ethical challenges that RIPs present from the motivation to combine competing interests, priorities and expectations of these diverse research partners. We claim that while critical perspectives on each of the partnership forms outlined above exist in the literature, the combination of industry, research and PPI actors in partnership in mental health research has not received sufficient scrutiny. Although the severity of the ethical issues that arise will depend on various factors, e.g. the nature of the study in question; the type of research partnership (i.e. research partnerships vary on strategies and may permit greater or lesser distance among partners ${ }^{8}{ }^{9}$ ) mental health studies relying on such partnership models are likely to present overlapping ethical problems and introduce challenges that affect research partners' relationship, research processes, and research output. We conclude that ethical guidance is needed to turn these challenges into best practices in mental health research.

\section{Ethical challenges in RIPS}

\section{Competing interests and priorities as barriers to the conduct of research}

Research partners may state a common shared goal of promoting improvements in mental health through the production of reliable and relevant evidence that leads to better treatments. However, they are also driven by different and often competing interests. We propose a crude summary of these interests here: Researchers are interested primarily in scientific progress and are more likely to be involved in studies that would advance their careers and reputation, such as studies that could be published in high impact journals ${ }^{10}{ }^{11}$. Industry funders tend to focus on interventions that have a high likelihood of translational success ${ }^{12}$; or that enhance basic knowledge required for advancement of the science relevant to drug / treatment discovery. Better care, if achieved, tends to be a by- product of commercial interest ${ }^{13}$. Finally, people with experience of mental health challenges tend to be interested in studies that provide safer and more effective interventions with fewer side effects ${ }^{12}$.

Competing interests, if not effectively managed, may cause internal ethical problems in RIPs; such as misunderstandings and conflicts among partners, which will affect the conduct of research. Conflicts may arise over the research agenda and methodology, interpretation and dissemination of the results, or even the overarching conceptual framework within which mental health research is undertaken. Indeed, one of the 
main difficulties in such research partnerships is that industry, academia and patients'/carers' agendas only partially overlap ${ }^{14}{ }^{15} 16$. As Crowe et al. have showed (2015), there is an important mismatch between the treatments evaluated by researchers and those that patients and clinicians want to see evaluated. In particular, the authors found that only a low proportion (2.6\%) of industry-funded registered trials studied the effects of the non-drug treatments and used non-drug comparators (such as drugs with psychological therapies for treating depression), despite the fact that non-drug interventions were preferred by patients. ${ }^{16}$ Similar results were also reported in a study carried out by Tallon and colleagues (2000). ${ }^{15}$ Those who have experienced mental distress and/or services first-hand frequently prioritise quality of life and are more interested in exploring strategies of managing mental health disorders from a holistic perspective and asking research questions on practical aspects of living, such as the nature of mental health services ${ }^{17}$. Moreover, they prefer social and psychological studies rather than biomedical ${ }^{18}$. Pharmaceutical companies, some researchers and national funders, in contrast, prioritise drug development.

To address potential and actual disagreements among research partners, the establishment of clear processes is necessary. However, managing conflicts in RIPs may be particularly challenging for two reasons. First, the substantial heterogeneity within two out of three of the entities brought together in collaboration (researchers and patient/carers) suggests that a straightforward process that can be shared by everyone involved cannot be easily identified. Mental health research is highly interdisciplinary; and mental health patient and carer communities are substantively diverse, particularly across different kinds of disorders. Interdisciplinarity and diversity can create challenges for mutual understanding between partners ${ }^{19}$. Conflicts arising from differing perspectives may occur among partners and within each partner group. For instance, patients diagnosed with autism may hold different views on how a study should proceed or on the roles they wish to hold/the activities they want to be involved in the project, as compared to carers ${ }^{20}$. Within the research group, psychologists and biological psychiatrists conducting research on autism comparing drug and psychological interventions may hold different a priori ideas about the value of the pharmacological and psychological therapy arms of the study.

A second reason for which managing disagreements within RIPs is challenging is due to the lack of clarity in how the diverse views of partners should be weighed. In the two RIP models outlined above the third partner is not explicitly in relationship with the other two primary partners, thereby further complicating a transparent articulation of processes to address competing priorities and interests. When such conflicts appear, there is a risk that the third entity's interests enter without proper process, to essentially adjudicate the conflict. For example, industry may be more interested in prioritising autism treatments that yield more commercial benefit. This may decide the direction of the research study, but it is hardly a good way to settle conflicting priorities in RIP research.

\section{Stereotype views as barriers to RIPs' stated goals}

By aiming to combine diverse forms of knowledge, skills and expertise, RIPs implicitly and explicitly introduce different roles and responsibilities to those assumed in conventional medical research models. In RIPs research partners are being required to develop and manage an entirely new research culture. However, the practical and ethical dimensions of such endeavour have been scarcely acknowledged by the national actors who require it.

Current guidelines aim to strengthen the role of lay people in research (i.e. PPI involvement as a requirement in research) but simply asserting the importance and value of PPI, does not itself overcome long held stigma about mental health service users ${ }^{21}$. As discussed in the PPI literature, people diagnosed with mental disorders are often assumed to be incapable of making reliable, rational judgments, and this undermines their role in research discussions ${ }^{22}{ }^{23}$. Academic researchers view themselves as holding scientific and objective views about research ${ }^{24}$ and consider any diversion from clinical methodology as impairing research quality ${ }^{21}$. As a consequence, researchers may refuse or ignore arguments against their supporting hypothesis ${ }^{25}$ or may even 
ask patients to be involved after the research questions have been decided to avoid 'over-involvement ${ }^{26}$. PPI stakeholders, on the other hand, argue that researchers follow specific research agendas aligned with their own academic interests and anticipations, and are unaware of their own biases ${ }^{27}$.

Stigmatising views about people with mental health conditions are also likely to exist within industry ${ }^{26}$. There is preliminary evidence to suggest that industry researchers do not view PPI involvement as an uncomplicated good, and that biased beliefs about the capacity of patients to be involved in research exist even outside mental health research. In Parsons et al's (2016) pilot study, some pharmaceutical industry professionals saw the primary role of people with lived experience of health conditions in research as that of research subjects ${ }^{28}$. Similar studies ${ }^{29} 30$ show inconsistent and fragmentated approaches to the involvement of people with firsthand experience of health services in industry-sponsored drug development research. This was due to real or perceived barriers by representatives of pharmaceutical companies, such as the need to report adverse events reported by patients, or the idea that direct interaction with people with first-hand experience of health services constitutes a regulatory violation ${ }^{31}$.

Preconceptions and stereotype views also exist regarding industry's role as research partner both within and outside research. Concerns and criticisms are often expressed by academic researchers ${ }^{32}{ }^{33}$ and patient groups $^{34} 35$ regarding pharmaceutical companies' manipulative role in selecting research priorities, managing the process of research and interfering with the interpretation and dissemination of results.

The existence of stereotype views and preconceptions that research partners may hold for each other regarding their moral integrity or ability to contribute to the quality of mental health research may work as barriers to meaningful collaboration and contradict RIPs' stated partnership goals. They also raise a more fundamental question about the justifications for RIPs in the first place, which have not currently been addressed in the research ethics literature. Reliance on diverse and interdependent kinds of knowledge, skills and expertise in mental health research is likely to raise barriers to effective collaborations and to the production of valuable knowledge; at what point are these problems more harmful than helpful to research? Are there any types of mental health research where such partnerships are not appropriate?

\section{Systemic power and control inequalities as barriers to fair partnerships}

In RIPs partners differ in their capacities, motivations and views about mental health research and their expectations about their roles and contribution ${ }^{16} 36{ }^{37}$. Differences about how a partnership should work in practice and decisions regarding roles and responsibilities are usually subject to negotiations among partners 49. Yet, in RIPs such negotiations are particularly challenging.

Hierarchies of knowledge and systemic power inequalities imbedded in the mental health context, may affect RIPs' dynamics ${ }^{38} 39$ and thus the processes and results of partners' negotiations. ${ }^{404142}$ According to evidence only few studies based on partnerships with industry, academia and people with first-hand experience of health services are deemed to meet standards of fairness ${ }^{1626}{ }^{43}$. Several authors ${ }^{44} 4527$ have pointed out that such partnerships are often structured in ways that favour researchers and/or funders more than people with first-hand experience of health services and preserve the status quo of scientific convention. For instance, PPI contributors may not be acknowledged as co-authors ${ }^{46}$; may receive no payment for their contributions ${ }^{47}$; or may lack power to influence the study once it has started. ${ }^{35}$ Similarly, mental health researchers may be forced to make several compromises that run serious professional and personal costs for them, which are masque by the idea of collaboration. ${ }^{48}$

Unfair practices regarding data sharing, intellectual property ${ }^{14} 16$ and remuneration in favour of those with greater control or authority are well documented in several partnership models and contexts ${ }^{49}$. Yet, in RIPs a distinct lack of guidance in how to manage partners' different needs and expectations regarding their roles and responsibilities in mental health research is obvious. As a consequence, RIP partners are engaged in a 
revolutionary experiment with little mandate to prevent unfair practices. In addition, the costs of such partnerships (e.g. waste of resources, professional and ideological compromises, etc.) are largely veiled by the ideals of RIP collaborations espoused by national actors. In order to keep 'normal' science going, RIPs may not only be ill-suited to identify and address unfairness but even be motivated to exaggerate existing power and control inequalities among mental health stakeholders.

\section{Lack of transparency in RIPs' policies and organisational structures as barriers to trustworthy partnerships}

In a conventional research model, academic researchers conduct and control the study with superficial or no involvement of other mental health stakeholders. In RIPs new formal relationships among mental health stakeholders are introduced where responsibilities, ownership and control are assumed to be, up to an extent, shared among the members of the partnership. In the two RIPs profiled above, a lack of clarity and a lack of transparency in partnership policies and the structure of obligations among the three 'partnering' entities are obvious. As a result, novel concerns may be raised about RIPs accountability at both partnership and public level. At best, the current situation on how such partnerships work, can be regarded as a move in a more inclusive direction. Yet, the process is far from fully worked out. For instance, although integrating measures for partnering with patients in new research partnership models represents a shift from disease-centred to patient-centred approaches to health care ${ }^{26}$, the problem of how to integrate patients' priorities into research agendas when the research is industry-sponsored, remains so far unresolved. Using the NIHR model as an example, it is not even clear that industry-sponsored research that is conducted within the NHS is required to integrate patient priorities and values - even though this is required for all other kinds of NIHR-supported research.

Well-known challenges regarding patient representation and diversity in mental health research add further complications regarding public accountability of RIPs and may introduce unreliability into the research process. Recent studies suggest that in new research models, researchers may favour PPI groups that are likely to share their views and legitimise their scientific endeavours ${ }^{35}$. Similar problems concern industry partners especially when RIPs involve patient organisations or groups. As the nature of the funding activities and the scale of drug companies investment in patient groups are not always transparent ${ }^{50}$, there is a risk that PPI partners may appear as representing patient views or advocating for people with mental illness ${ }^{51}{ }^{52}$ while in reality they may promote marketing interests ${ }^{53}$ or even encourage therapies with problematic clinical profiles and cost effectiveness. ${ }^{54}$

Lack of clear public communication strategies regarding partnership policies and governance structures may undermine the trustworthiness of RIPs and the potential for ethical collaborations. This will arguably make for ongoing problems in many such partnerships, but it is a particular problem in mental health research. Relations among people with lived experience of mental illness, researchers and industry have long been fraught by accusations of unethical practices ${ }^{53}$, which continue to this day, and carry enormous personal and economic consequences. Given the turbulent and ethically problematic history of relations between the profession of psychiatry and psychiatric 'users', the involvement of people with lived experience of mental health difficulties in research could be seen to carry distinctive ethical weight. The addition of industry as the third 'partner' in a mental health research 'collaboration' adds further fragility to an already vulnerable enterprise. Mental health research relies heavily on industry funding. It is thus essential that ethical principles that would support worthwhile projects and trustworthy RIPs are guiding the relationships of RIP partners and are clearly communicated to the public.

\section{A need for ethical guidance}

Despite current enthusiasm about their potential "to transform the landscape of mental health research" RIPs present ethical challenges, which are veiled by the ideals of RIP collaborations. Whether this is due to a 
genuine desire for revolution on the part of senior health policy makers and funders, or a lack of foresight while appeasing public sentiment, the result is that RIP partners have a distinct lack of guidance in how to manage the costs of participating in a revolutionary experiment. We suggest that ethical guidance is essential to support successful partnerships and promote fair and ethical relationships among partners in mental health research.

An important step towards this aim is to develop an ethical account of what constitutes ethical partnership with industry, academia and people with first-hand experience in mental health illness. To establish ethically credible RIPs, RIP partners should rely on firm ethical values.

To help RIP partners in taking actual steps to ensure that best practices are followed when planning and forming such partnerships and conducting research, it is important that ethical guidance is informed by evidence on the challenges that RIPs encounter in different mental health research projects. Associated drawbacks of RIPs, such as time, cost and use of limited resources, should not be disproportionate relative to potential benefits of the research. Evidence-based and clear justifications about the value and impact of RIPs in different types of mental health research could also help correct, false presumptions and stereotypes that partners may hold for each other and appreciate the importance of creating the right platforms where they all can meaningfully work together ${ }^{55} 18$.

Ethical guidance should also provide problem-solving strategies and discuss what form of 'joint reasoning' is appropriate in different types of research partnerships so that partners are guided by a clear and convincing account of how shared decision-making should look like in these settings. Although it is possible (and perhaps more appropriate) to advocate for different solutions in different research settings and kinds of mental health research (as these ethical problems may arise differently in different mental health research projects), it is important to ensure that RIP partners rely on transparent and clear procedures that ensure mutual understanding and promote fairness. Finally, current institutional oversight paradigms need to be adapted to accommodate RIPs and address the issues discussed above.

\section{Conclusion}

The aim of this paper was to consider the ethical challenges that arise when researchers, industry and individuals with experience of mental health illness are involved as partners in mental health research. We discussed barriers in knowledge production, unfair partner relationships, conflicts in decision making and lack of clear public communication. We do not suggest that RIPs are a misguided or a wrong effort; rather that the practical and ethical work of RIPs is severely undermined and risky without a coherent account of the concept of RIP collaboration and its underlying justifications. The future of mental health research will rely increasingly on interactions between academia, industry and individuals with first-hand experience of mental illness. Given that these problems are not addressed by current guidelines, we suggest that a robust ethical account is needed to properly substantiate the value of such research partnerships, to provide practical and ethical guidance on potential conflicts and to facilitate productive collaborative research. We view the preceding analysis as the necessary groundwork to identify the systematic processes that can enable good RIP partnerships and valuable research outputs.

\section{Conflicts of interest statement}

We have no conflicts of interest to report. 


\section{Funding details}

SL and IS are supported by the Wellcome Centre for Ethics and Humanities, which is supported by core funding from the Wellcome Trust [203132/Z/16/Z] in addition to the NIHR Oxford Health Biomedical Research Centre [IS-BRC-1215-20005]. IS is additionally supported by the Wellcome Trust [104825/Z/14/Z].

\section{References}

${ }^{1}$ Innovative Medicines Initiative. IMI pool of patient experts. https://www.imi.europa.eu/getinvolved/patients/imi-pool-patient-experts.

${ }^{2}$ National Institute for Health Research. Partners and Industry. https://www.nihr.ac.uk/partners-andindustry/).

${ }^{3}$ National Institute for Health Research. Make a strong application. https://www.nihr.ac.uk/researchers/apply-for-funding/how-to-apply-for-project-funding/make-a-strongapplication.htm).

${ }^{4}$ National Institute for Health Research. Run your study in the NHS. https://www.nihr.ac.uk/partners-andindustry/industry/run-your-study-in-the-nhs/

${ }^{5}$ National Institute for Health Research. https://industryroutemap.netlify.com/story_html5.html?lms=1

${ }^{6}$ Schachar R, Ickowicz A. Funding for mental health research: looking ahead. J Can Acad Child Adolesc Psychiatry. 2014;23(2):84-85.

${ }^{7}$ National Institute for Health Research. It's simple: https://www.nihr.ac.uk/blog/its-simple-mental-healthresearch-collaboration-will-improve-lives/11036

${ }^{8}$ Carrick R, Mitchell A, Lloyd K. User involvement in research: power and compromise. J. Community. Appl. Soc. Psychol 2001; 11: 217-225

${ }^{9}$ Trivedi P, Wykes T. From passive subjects to equal partners: Qualitative review of user involvement in research. British Journal of Psychiatry 2002;181(6):468-472.

${ }^{10}$ Rothenburg ML, Johnson DH: Conflict of interest, conflicting interests, and effective collaboration between academia and industry on preclinical and clinical cancer research. JAMA Oncol 2017;3:1621-1622.

${ }^{11}$ White-Cooper S, Dawkins N U, Kamin S L, Anderson L A. Community-Institutional Partnerships: Understanding Trust Among Partners. Health Education \& Behavior 2009; 36(2), 334-347.

${ }^{12}$ Mader LB, Harris T, Kläger S, Wilkinson IB, Hiemstra TF. Inverting the patient involvement paradigm: defining patient led research. Res Involv Engagem. 2018;4:21.

${ }^{13}$ Tierney WM, Meslin EM, Kroenke K. Industry Support of Medical Research: Important Opportunity or Treacherous Pitfall?. J Gen Intern Med. 2016;31(2):228-233.

${ }^{14}$ Elwyn G, Miron-Shatz T. Deliberation before determination: the definition and evaluation of good decision making. Health Expectations 2010; 13: 139-147.

${ }^{15}$ Tallon, D. Relation between agendas of the research community and the research consumer. The Lancet 2002; 355 (9220): 2037- 2040.

${ }^{16}$ Crowe S, Fenton M, Hall M, Cowan K, Chalmers I. Patients', clinicians' and the research communities' priorities for treatment research: there is an important mismatch [published correction appears in Res Involv Engagem. 2015 Dec 23; 1:14]. Res Involv Engagem 2015; 1:2.

${ }^{17}$ Department of Health. Strategic reviews of research and development mental health report appendices. 2002, Available from: http://www.doh.gov.uk/research/documents/mhfinalappendicesc.pdf [Google Scholar] 
${ }^{18}$ Rose D. Participatory research: Real or imagined. Social Psychiatry and Psychiatric Epidemiology: The International Journal for Research in Social and Genetic Epidemiology and Mental Health Services 2018; 53(8): 765-771.

${ }^{19}$ Hanley B, Bradburn J, Barnes M, Evans C, Goodare H, Kelson M. Involving the public in NHS,Public Health and Social Care Research: Briefing notes for researchers.(second edition). Eastleigh: INVOLVESupport Unit' 2004.

${ }^{20}$ NHS Good Practice Guidance for involving people with experience of mental health problems in research 2013, Available: http://www.rds-

sw.nihr.ac.uk/documents/NIHR_MHRN_Involving_Mental_Health_Problems_Research2013.pdf

${ }^{21}$ Lambert N, Carr, S. 'Outside the Original Remit': Co-production in UK mental health research, lessons from the field. Int J Mental Health Nurs 2018; 27: 1273-1281.

${ }^{22}$ Rose D. Having a diagnosis is a qualification for the job. British Medical Journal 2003; 326: 1331.

${ }^{23}$ Beresford P. User involvement in research and evaluation: liberation or regulation? Social Policy \& Society 2002; 1(2) 95-105.

${ }^{24}$ Macran S, Ross H, Hardy G, Shapiro D. The importance of considering clients' perspectives in psychotherapy research. Journal of Mental Health 1999; 8(4), 325-337.

${ }^{25}$ Faulkner A, Carr S, Gould D, et al. 'Dignity and respect': An example of service user leadership and coproduction in mental health research. Health Expectations. 2019; 00:1-10.

${ }^{26}$ du Plessis D, Sake J-K, Halling K, Morgan J, Georgieva A, Bertelsen N. Patient Centricity and Pharmaceutical Companies: Is It Feasible? Therapeutic Innovation \& Regulatory Science 2017; 51(4): 460-467.

${ }^{27}$ Beresford, P. It's Our Lives: a Short Theory of Knowledge, Distance and Experience. in association with Shaping Our Lives. London: Citizen Press; 2003.

${ }^{28}$ Parsons S, Starling B, Mullan-Jensen C, Tham SG, Warner K, Wever K. What do pharmaceutical industry professionals in Europe believe about involving patients and the public in research and development of medicines? A qualitative interview study. BMJ Open 2016; 6:e008928.

${ }^{29}$ Hoos A, Anderson J, Boutin M, et al. Partnering with patients in the development and lifecycle of medicines: a call for action. Ther Innov Regul Sci 2015; 49:929-939.

${ }^{30}$ Blackburn S, McLachlan S, Jowett $S$ et al. The extent, quality and impact of patient and public involvement in primary care research: a mixed methods study. Res Involv Engagem 2018; 4: 16.

${ }^{31}$ Lowe MM, Blaser DA, Cone L, et al. Increasing Patient Involvement in Drug Development. Value Health. 2016;19(6): 869-878.

${ }^{32}$ Capps B. Can a good tree bring forth evil fruit? The funding of medical research by industry. Br Med Bull. 2016;118(1):5-15.

${ }^{33}$ Stone VJ. American Association of University Professors. Recommended Principles to Guide AcademyIndustry Relationships. Champaign, IL: University of Illinois Press; 2014.

${ }^{34}$ Carrier F, Banayan D, Boley R, Karnik N. Ethical challenges in developing drugs for psychiatric disorders. Prog. Neurobiol 2017; 152: 58-69

${ }^{35}$ Russell G, Starr S, Elphick C, Rodogno R, Singh, I. Selective patient and public involvement: The promise and perils of pharmaceutical intervention for autism. Health Expect. 2018; 21: 466- 473.

${ }^{36}$ Green G. Power to the people: To what extent has public involvement in applied health research achieved this? Research Involvement and Engagement 2016; 2:n.pag

${ }^{37}$ Filipe A, Renedo A, Marston C. The co-production of what? Knowledge, values, and social relations in health care. PLoS Biol. 2017; 3;15(5):e2001403. 
${ }^{38}$ Tabriz AA, Flocke SA, Shires D, et al. Logic model framework for considering the inputs, processes and outcomes of a healthcare organisation-research partnership. BMJ Quality \& Safety; 2019. Published Online First: 11 December 2019. doi: 10.1136/bmjqs-2019-010059

${ }^{39}$ Lignou S, Capitao L, Hamer-Hunt JM, Singh I. Co-Production: An Ethical Model for Mental Health Research?, The American Journal of Bioethics 2019; 19:8, 49-51

${ }^{40}$ Mason J. Qualitative researching. London: Sage Publications; 2002.

${ }^{41}$ Greenhalgh T, Hinton L, Finlay T, et al. Frameworks for supporting patient and public involvement in research: Systematic review and co-design pilot. Health Expect. 2019; 22: 785- 801.

${ }^{42}$ Wallcraft J, Amering M, Freidin J et al. Partnerships for better mental health worldwide: WPA recommendations on best practices in working with service users and family carers. World Psychiatry 2011; 10: 229- 36.

${ }^{43}$ Fisher M. The role of service users in problem formulation and technical aspects of social research. Social Work Education 2002; 21: 305-312.

${ }^{44}$ Beresford P. From 'other' to involved: user involvement in research: an emerging paradigm, Nordic Social Work Research 2013; 3(2): 139-148,

${ }^{45}$ Faulkner, A. The ethics of survivor research: Guidelines for the ethical conduct of survivor research. Bristol: Policy Press in association with the Joseph Rowntree Foundation; 2004.

${ }^{46}$ Resnik DB, Elliott KC, Miller AK. A framework for addressing ethical issues in citizen science, Environmental Science \& Policy 2015; 54: 475-481.

${ }^{47}$ Riesch H, Potter C, Citizen science as seen by scientists: methodological, ethical, and epistemological dimensions. Public Underst. Sci 2014; 23: 107- 120.

${ }^{48}$ Oliver K, Kothari A, Mays N. The dark side of coproduction: do the costs outweigh the benefits for health research? Health Res Policy Syst 2019;17(1):33.

${ }^{49}$ Fransman J, Newman, Kate and Cornish, Hilary. Rethinking Research Partnerships: Discussion Guide and Toolkit. Christian Aid; 2017

${ }^{50}$ EFPIA. EFPIA code of practice on relationships between the pharmaceutical industry and patient organisations, 2011.

${ }^{51}$ McCoy MS, Carniol M, Chockley K, Urwin JW, Emanuel EJ, Schmidt H. Conflicts of interest for patientadvocacy organizations. N Engl J Med 2017;376:880-5.

${ }^{52}$ Rose SL, Highland J, Karafa MT, Joffe S. Patient advocacy organizations, industry funding, and conflicts of interest. JAMA Intern Med 2017; 177:344 -50.

${ }^{53}$ Ozieranski $\mathrm{P}$, Rickard E, Mulinari S. Exposing drug industry funding of UK patient organisations BMJ 2019; 365 :1806

${ }^{54}$ Hughes D, Williams-Jones B. Coalition Priorite Cancer and the pharmaceutical industry in Quebec: conflicts of interest in the reimbursement of expensive cancer drugs? Healthc Policy 2013; 9:52-64.

${ }^{55}$ Pestoff V. Collective Action and the Sustainability of Co-Production. Public Management Review 2013; 16 (3): 383-401. 